\title{
A BDF2 energy stable scheme for the binary fluid-surfactant hydrodynamic model
}

\author{
Yuzhe Qin ${ }^{1}$, Rui Chen ${ }^{2}$, and Zhengru Zhang ${ }^{1}$ \\ ${ }^{1}$ Beijing Normal University \\ ${ }^{2}$ Beijing University of Posts and Telecommunications
}

November 13, 2020

\begin{abstract}
A second-order time stepping scheme is developed for the binary fluid-surfactant phase field model coupled with hydrodynamics by using the scalar auxiliary variable approach and pressure correction method. The free energy contains a double-well potential, a nonlinear coupling entropy and a Flory-Huggins potential. By introducing one scalar auxiliary variable, the system is transformed into an equivalent form so that the nonlinear terms can be treated semi-explicitly. The scheme is linear and decoupled; thus, they can be solved eciently. We further prove that the semidiscretized scheme in time is unconditionally energy stable. Numerical experiments are performed to validate the accuracy and energy stability of the proposed scheme.
\end{abstract}

\section{Hosted file}

afterrunse2.pdf available at https://authorea.com/users/375503/articles/492721-a-bdf2energy-stable-scheme-for-the-binary-fluid-surfactant-hydrodynamic-model 